\section{Cureus}

Received 06/03/2019

Review began 06/05/2019

Review ended 06/13/2019

Published 06/30/2019

\section{(c) Copyright 2019}

Lee et al. This is an open access article distributed under the terms of the Creative Commons Attribution License CC-BY 3.0., which permits unrestricted use, distribution, and reproduction in any medium, provided the original author and source are credited.

\title{
Extensive Unpredictable Pancreas Cancer Inter-fraction Motion: A Case Report
}

Sangjune L. Lee ${ }^{1}$, Michael Velec ${ }^{2}$, Pablo Munoz-Schuffenegger ${ }^{3}$, Teo Stanescu ${ }^{4}$, Laura Dawson $^{5}$

1. Radiation Oncology, University of Toronto, Toronto, CAN 2. Radiation Medicine, Princess Margaret Hospital/University Health Network, Toronto, CAN 3. Hematology - Oncology, Pontifical Catholic University of Chile, Santiago, CHL 4. Medical Physics, Princess Margaret Cancer Centre, Toronto, CAN 5. Radiation Oncology, Princess Margaret Cancer Centre, Toronto, CAN

$\square$ Corresponding author: Laura Dawson, laura.dawson@rmp.uhn.ca

Disclosures can be found in Additional Information at the end of the article

\section{Abstract}

We present a case of locally advanced pancreatic cancer with duodenal invasion treated with consolidative chemoradiation, where extensive unpredictable interfraction motion was observed. Initially, two attempts were made to treat with volumetric modulated arc therapy technique. However, due to substantial interfractional motion of the pancreatic head mass relative to the regional nodal areas, the patient was eventually replanned and treated with a four-field box technique. This case highlights the difficulty in delivering conformal radiation to the pancreas and quantifies the movement of the target, the adjacent biliary stent, and regional nodes.

Categories: Radiation Oncology, Gastroenterology, Oncology

Keywords: pancreatic cancer, conformal radiation, four-field box, organ motion

\section{Introduction}

Patients with pancreatic cancer have a five-year overall survival of $6 \%$, and only $15 \%$ of presenting cases are resectable [1]. Effective and safe radiation therapy delivery to the pancreas is challenging due to the proximity of numerous moving, dose-limiting organs at risk [2]. We present a case of locally advanced pancreatic cancer treated with consolidative chemoradiation, where extensive unpredictable inter-fraction motion was observed.

\section{Case Presentation}

A 75-year-old woman with a gastrointestinal stromal tumor resected eight years prior presented with a 44-mm mass in the head of the pancreas encasing the superior mesenteric artery (SMA), a 13-mm node at the proximal SMA, invasion of the duodenum, and biliary obstruction on CT. An endoscopic retrograde cholangiopancreatography (ERCP), metal stent insertion and brushings confirmed an adenocarcinoma. She then received nine cycles of fluorouracil, leucovorin, irinotecan and oxaliplatin (FOLFIRINOX) over eight months. After seven months of chemotherapy, CT showed an excellent response with a residual $30 \mathrm{~mm}$ hypoattenuating mass. Consolidative concurrent chemoradiation, $52.5 \mathrm{~Gy}$ in 30 daily fractions, with biweekly low dose gemcitabine was offered [3].

At simulation, a helical exhale breath-hold CT with intravenous and oral contrast and a 4DCT were obtained. A 10-mm gross target volume (GTV) expansion plus initially involved nodes were combined with regional nodal regions to create clinical target volumes (CTVs) on helical, 


\section{Cureus}

inhale and exhale CT images [4]. The combined CTVs formed an internal target volume (ITV) that was isotropically expanded by $5 \mathrm{~mm}$ to create the planning target volume (PTV). The first volumetric modulated arc therapy (VMAT) plan (Pinnacle v9.6, Philips Medical Systems, Madison, WI) was created and delivered in free breathing (Figure 1 and Figure 2).

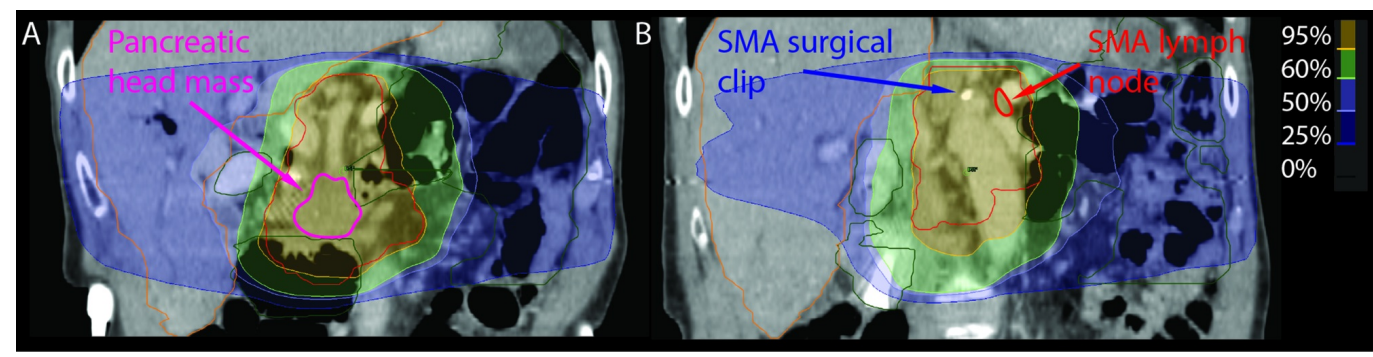

\section{FIGURE 1: Coronal views of the isodose distribution for the original VMAT plan.}

The pancreatic head mass $(A)$ is located anteriorly and inferiorly to the SMA node (B). A metal clip from a previous surgery, superior to the take-off of the SMA, served as a fiducial for the SMA node on CBCT.

SMA: Superior mesenteric artery; VMAT: Volumetric modulated arc therapy; СВCT: Cone-beam computed tomography.

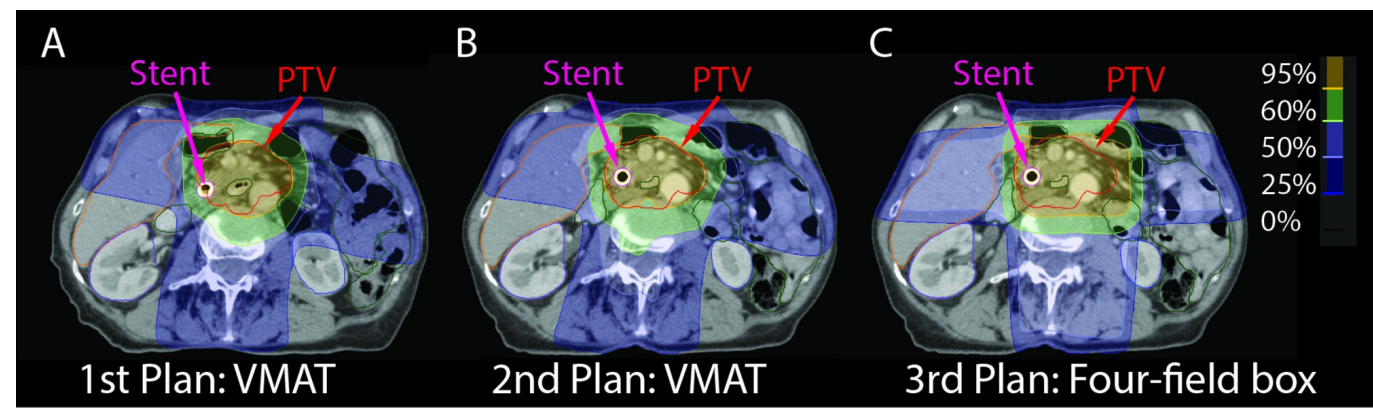

\section{FIGURE 2: Axial views of the isodose distribution for each of the radiation plans.}

First plan: VMAT (A), second plan: VMAT (B), third plan: FFB (C). Plans were scaled to the original prescription of $52.5 \mathrm{~Gy}$ in 30 fractions for comparison.

FFB: Four-field box; VMAT: Volumetric modulated arc therapy; PTV: Planning target volume.

Daily image-guidance was based on cone-beam CT (CBCT) with a bone match, followed by a review of the stent position as it was adherent to the pancreatic head. CBCT showed wide stent variations in position and fluctuations in the volume of bowel gas despite the patient's adherence to our dietary preparation instructions of no food for two hours prior to treatment. A repeat CT simulation for replanning on fraction 4 was ordered to account for the shift in the stent and pancreas, relative to the stable SMA. The GTV could be covered with the original plan by shifting the patient position laterally; however, this shift led to under-coverage of the SMA nodal CTV. A surgical clip from eight years prior, superior to the SMA root and less than $20 \mathrm{~mm}$ right of the suspicious SMA node, was identified as a surrogate for the SMA node on CBCT. A 


\section{Cureus}

second VMAT plan, with a 6-mm isotropic ITV to PTV expansion, was initiated on fraction 6. However, the changes in stent position by up to $30 \mathrm{~mm}$ continued to be observed (Figure 3).

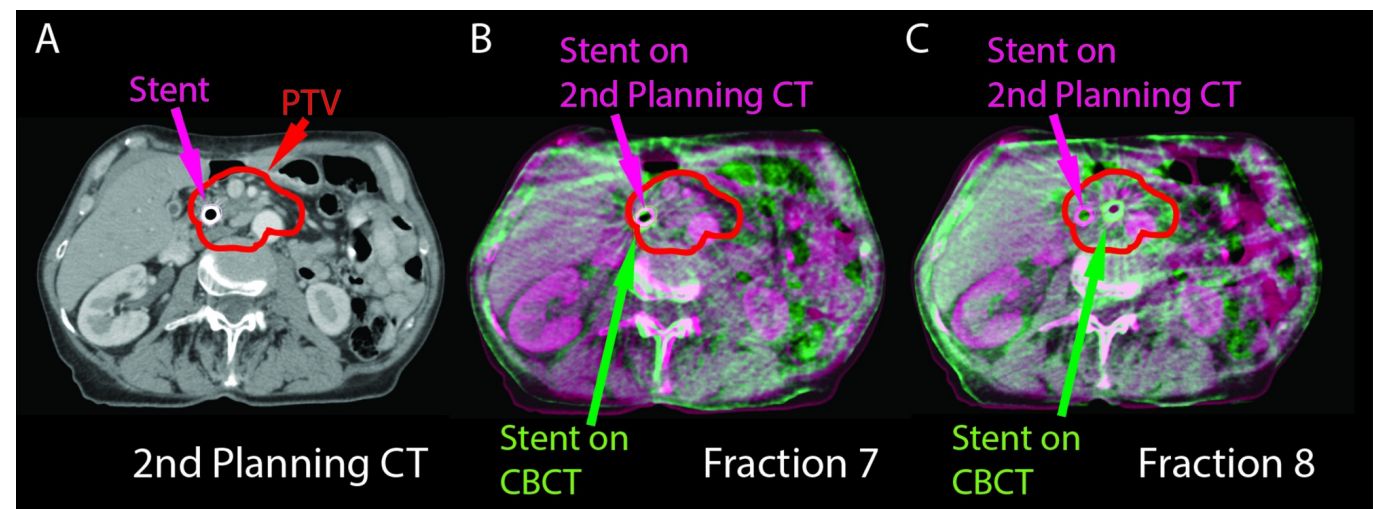

\section{FIGURE 3: CT and CBCT fusions.}

Second planning CT (A) and fused planning CT with CBCTs are shown (B-C). The planning CT is in purple and the CBCT is in green. Although the stents were aligned on fraction 7 (B), on fraction 8 the stent moved $20 \mathrm{~mm}$ medially with maintained vertebral body alignment (C).

PTV: Planning target volume; СBCT: Cone-beam computed tomography.

To investigate possible intra-fractional motion, the patient underwent MRI on fraction 14 with 2D-cine acquisition through the target, an 8-mm slice thickness, in-plane resolution of 1 $\mathrm{mm}$ and a temporal resolution of $3 \mathrm{~Hz}$. Pancreas' respiratory motion was approximately $10 \mathrm{~mm}$ superior-inferior, $8 \mathrm{~mm}$ lateral, and $5 \mathrm{~mm}$ anterior-posterior. Inhale breath-hold MR images taken 30 minutes apart showed less than $10 \mathrm{~mm}$ stent motion. Overall, intra-fractional motion was less than inter-fractional motion. Due to concern of the compromised nodal CTV, the patient was replanned once again with a 'robust' four-field box (FFB) technique using the second planning CT starting on fraction 17. On this third plan, the ITV to PTV expansions were $7 \mathrm{~mm}$ anterior-posterior, $7 \mathrm{~mm}$ right, $17 \mathrm{~mm}$ left, and $7 \mathrm{~mm}$ superior-inferior. These expansions were specified to cover the inter-fraction motion seen on previous fractions.

Organ-at-risk (OAR) doses were increased (Figure 4) with the FFB plan, but the entire CTV remained within the high dose region for the remaining fractions. All planning objectives and OAR constraints were met including PTV D99\% >=95\%, PTV D0 cc < 105\%, liver mean < 30 Gy, liver V30 Gy < 60\%, bilateral kidneys mean < 18 Gy, bilateral kidneys V22.5 Gy < 33\%, duodenum D0.5 cc < 100\%, and spinal canal D0cc $<45$ Gy. The mid-stent position on daily CBCT was measured relative to the stable L2 vertebral spinous process. The pancreatic head mass location, inferred by the mid-stent position, varied substantially compared to the SMA node, inferred by the adjacent surgical clip, over all fractions (Figure 5 and Figure 6). 


\section{Cureus}

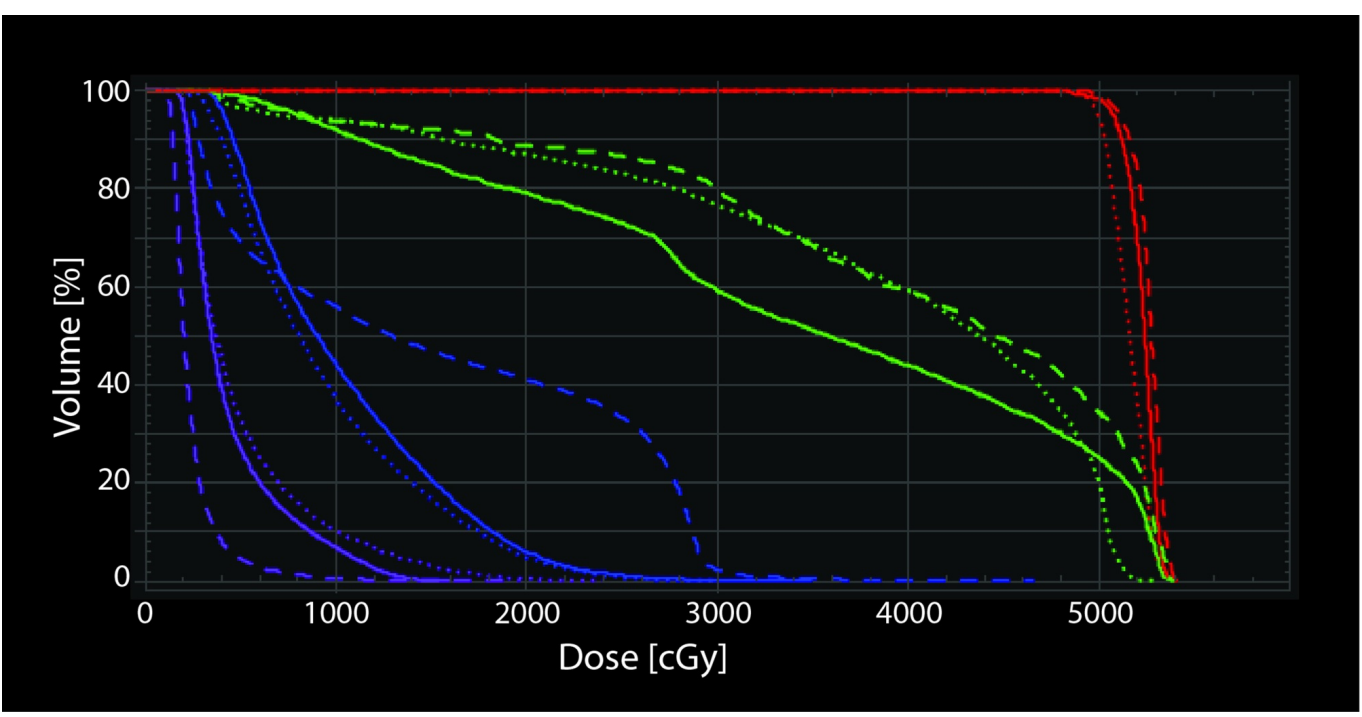

\section{FIGURE 4: Dose-volume histogram for the three treatment}

\section{plans used to treat this patient.}

This assumes all 30 fractions were delivered with each plan. First plan with VMAT (solid line), second plan with VMAT (dotted line), and third plan with FFB (dashed line) are shown with prescriptions set to $52.5 \mathrm{~Gy}$ in 30 fractions for comparison. PTV (red), duodenum (green), left kidney (blue) and right kidney (purple) are shown. FFB plan has higher doses to the organs at risk, except for the right kidney.

PTV: Planning target volume; СBCT: Cone-beam computed tomography; FFB: Four-field box; VMAT: Volumetric modulated arc therapy. 


\section{Cureus}

A

Left/Right

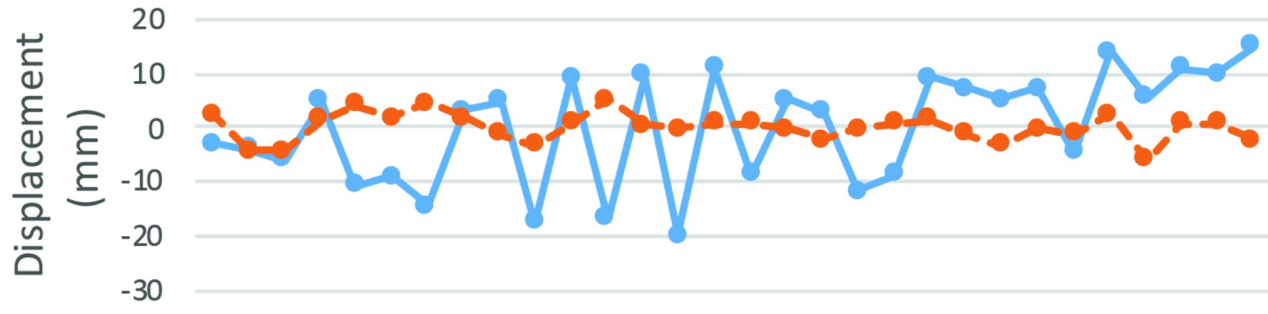

B

Anteror/Posterior

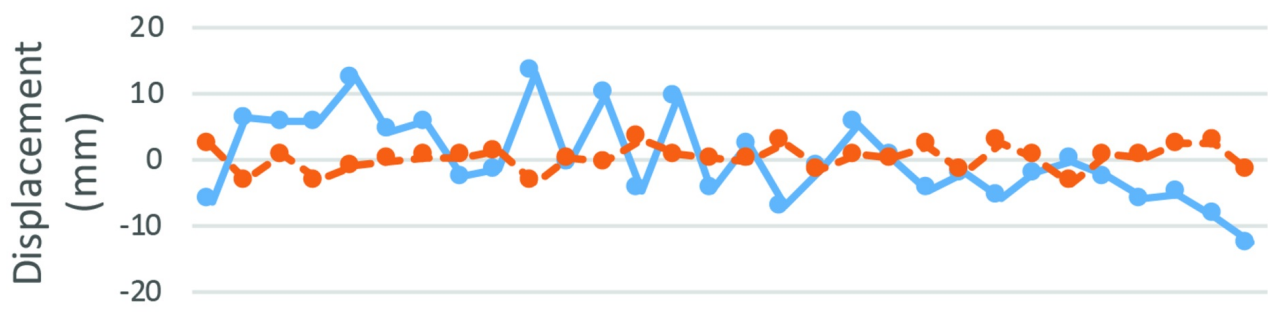

C

Superior/Inferior

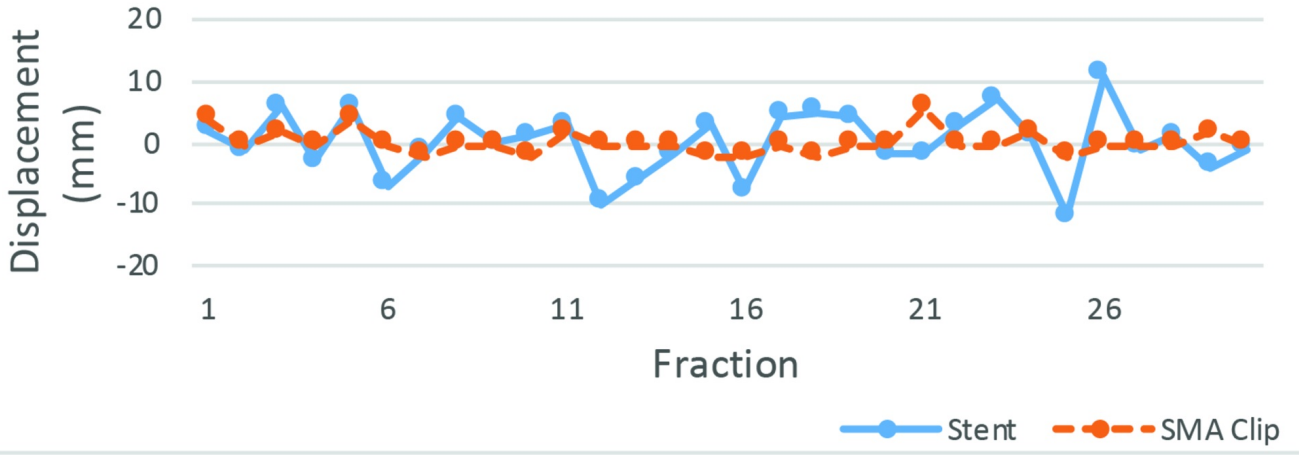

FIGURE 5: Stent and SMA clip displacement relative to their respective mean positions in three vector directions.

Left/right (A), anterior/posterior (B), and superior/inferior (C).

SMA: Superior mesenteric artery. 


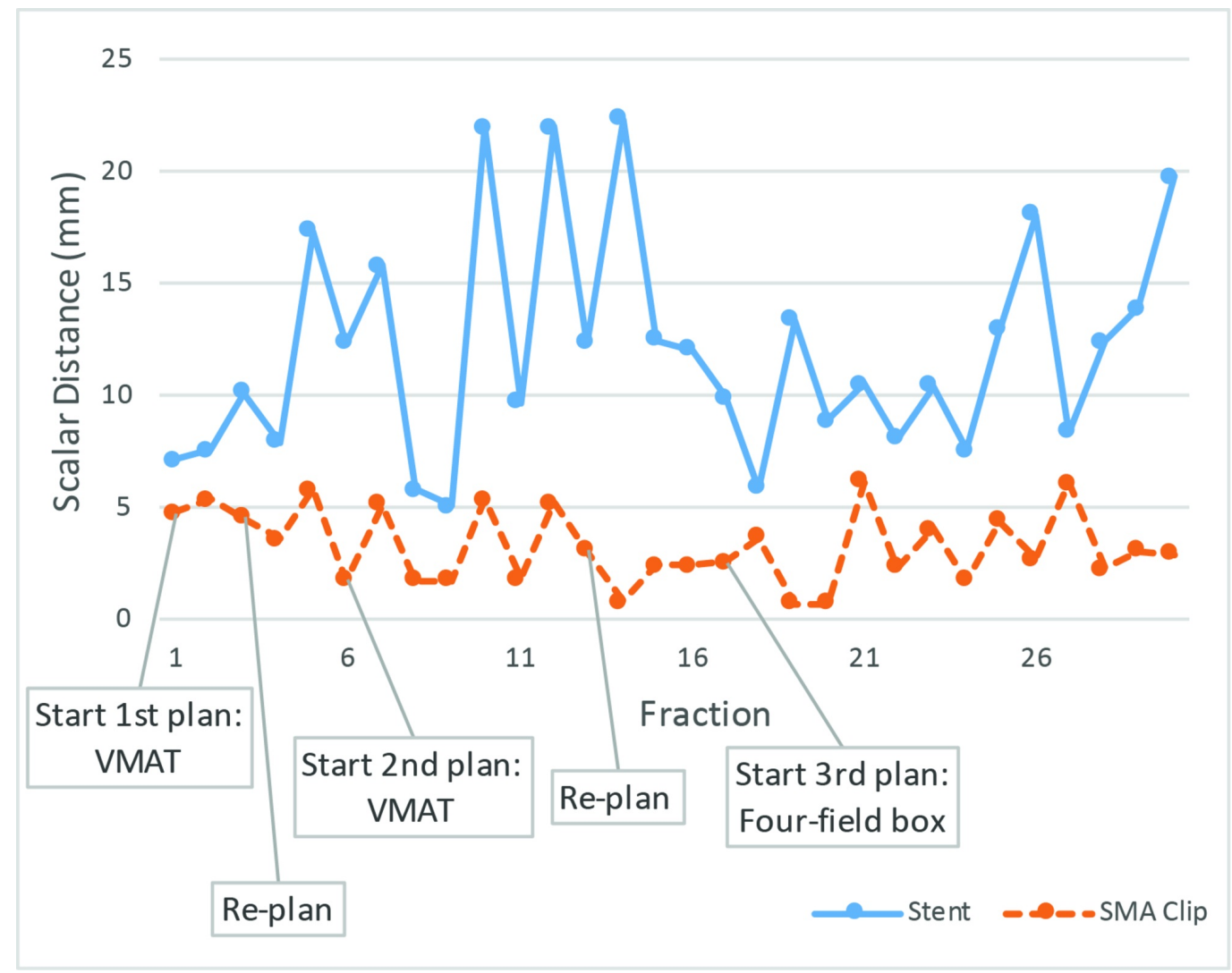

FIGURE 6: Stent and SMA clip scalar distance relative to their respective mean positions.

SMA: Superior mesenteric artery; VMAT: Volumetric modulated arc therapy.

The patient developed grade 2 thrombocytopenia and neutropenia, and gemcitabine was held for the second half of treatment. She also developed grade 2 fatigue, anorexia, and diarrhea which resolved before the completion of treatment. She remained clinically well six months after treatment, with no progression on follow-up CT.

\section{Discussion}

We report a case of locally advanced pancreatic cancer originally planned to be treated with consolidative chemoradiation therapy with VMAT technique. SBRT was avoided due to duodenal invasion on presentation [5]. However, due to substantial inter-fractional motion, the patient was eventually treated with an FFB technique, albeit with higher doses to adjacent organs at risk.

Compared to FFB, conformal techniques have resulted in decreased toxicity, however, they are more sensitive to geometric uncertainties [6,7]. Although the biliary stent is superior to bony anatomy, it is not a perfect surrogate for the GTV. Differences greater than $5 \mathrm{~mm}$ between motion of GTV and stent have been observed and therefore a stent should not be relied on as the sole surrogate in highly conformal plans [8,9]. Although metal seed fiducial markers around the pancreatic tumour can help localize the primary GTV, it is an invasive procedure, and does not help localize nodal CTVs, which may move discordantly compared to the primary CTV [10]. Even if the pancreas CTV may be localized, this case shows that shifting to cover pancreas CTV would result in under coverage of the SMA node. 
Studies suggest improved overall survival after treating pancreatic cancer with biologically effective doses above 70 Gy [11]. However, inter-fraction motion to the degree observed in this case makes it difficult to deliver higher doses safely unless online daily replanning is used to ensure proper coverage of both the primary and nodal CTV while avoiding critical OARs such as the duodenum. Changes in abdominal anatomy can be detected with daily CT or MR. Preliminary data show excellent outcomes with dose escalation and MRI-guided radiation therapy. Fast daily online adaptive workflows have been developed and daily replanning should account for discordant changes in primary and nodal CTVs and regional OARs, as observed in this case [12-14]. The potential for MRI guidance to improve clinical results is high but requires validation in larger comparative studies.

\section{Conclusions}

In this case report, we presented a woman with a locally advanced pancreatic cancer with a suspicious SMA node and duodenal invasion. Due to her clinical features, SBRT was not an option and therefore we attempted to treat with a conformal VMAT plan. However, because of extensive unpredictable inter-fraction motion, with greater movement at the pancreatic head mass relative to the stable proximal SMA node, the patient was replanned and treated with a four-field box technique. This case highlights the difficulty in delivering conformal radiation while maintaining adequate doses to both the regional nodes and pancreatic head.

\section{Additional Information}

\section{Disclosures}

Human subjects: Consent was obtained by all participants in this study. Conflicts of interest: In compliance with the ICMJE uniform disclosure form, all authors declare the following: Payment/services info: All authors have declared that no financial support was received from any organization for the submitted work. Financial relationships: All authors have declared that they have no financial relationships at present or within the previous three years with any organizations that might have an interest in the submitted work. Other relationships: All authors have declared that there are no other relationships or activities that could appear to have influenced the submitted work.

\section{Acknowledgements}

The authors would like to acknowledge Diana Lee for her notes on the patient setup.

\section{References}

1. Ilic M, Ilic I: Epidemiology of pancreatic cancer. World J Gastroenterol. 2016, 22:9694-9705. 10.3748/wjg.v22.i44.9694

2. Hammel P, Huguet F, Laethem J-L van, et al.: Effect of chemoradiotherapy vs chemotherapy on survival in patients with locally advanced pancreatic cancer controlled after 4 months of gemcitabine with or without erlotinib: the LAP07 randomized clinical trial. JAMA. 2016, 315:1844-1853. 10.1001/jama.2016.4324

3. Youl M, Hashem S, Brade A, et al.: Induction gemcitabine plus concurrent gemcitabine and radiotherapy for locally advanced unresectable or resected pancreatic cancer. Clin Oncol. 2014, 26:203-209. 10.1016/j.clon.2014.01.003

4. Caravatta L, Sallustio G, Pacelli F, et al.: Clinical target volume delineation including elective nodal irradiation in preoperative and definitive radiotherapy of pancreatic cancer. Radiat Oncol. 2012, 7:86. 10.1186/1748-717X-7-86

5. Balaban EP, Mangu PB, Khorana AA, et al.: Locally advanced, unresectable pancreatic cancer: American Society of Clinical Oncology Clinical Practice Guideline. J Clin Oncol. 2016, 34:26542668. 10.1200/JCO.2016.67.5561

6. Higgins PD, Sohn JW, Fine RM, Schell MC: Three-dimensional conformal pancreas treatment: 
comparison of four- to six-field techniques. Int J Radiat Oncol. 1995, 31:605-609.

10.1016/0360-3016(94)00380-4

7. Prasad S, Cambridge L, Huguet F, et al.: Intensity modulated radiation therapy reduces gastrointestinal toxicity in locally advanced pancreas cancer. Pract Radiat Oncol. 2016, 6:7885. 10.1016/j.prro.2015.09.006

8. Chu K-Y, Eccles CL, Brunner TB: Endobiliary stent position changes during external-beam radiotherapy. J Med Imaging Radiat Sci. 2015, 46:57-64. 10.1016/j.jmir.2014.08.004

9. van der Horst A, Lens E, Wognum S, de Jong R, van Hooft JE, van Tienhoven G, Bel A: Limited role for biliary stent as surrogate fiducial marker in pancreatic cancer: stent and intratumoral fiducials compared. Int J Radiat Oncol Biol Phys. 2014, 89:641-648. 10.1016/j.ijrobp.2014.03.029

10. Minn AY, Schellenberg D, Maxim P, et al.: Pancreatic tumor motion on a single planning 4DCT does not correlate with intrafraction tumor motion during treatment. Am J Clin Oncol. 2009, 32:364-368. 10.1097/COC.0b013e31818da9e0

11. Krishnan S, Chadha AS, Suh Y, et al.: Focal radiation therapy dose escalation improves overall survival in locally advanced pancreatic cancer patients receiving induction chemotherapy and consolidative chemoradiation. Int J Radiat Oncol Biol Phys. 2016, 94:755-765. 10.1016/j.ijrobp.2015.12.003

12. Bohoudi O, Bruynzeel AME, Senan S, Cuijpers JP, Slotman BJ, Lagerwaard FJ, Palacios MA: Fast and robust online adaptive planning in stereotactic MR-guided adaptive radiation therapy (SMART) for pancreatic cancer. Radiother Oncol. 2017, 125:439-444. 10.1016/j.radonc.2017.07.028

13. Rudra S, Jiang N, Rosenberg SA, et al.: Using adaptive magnetic resonance image-guided radiation therapy for treatment of inoperable pancreatic cancer. Cancer Med. 2019, 8:21232132. 10.1002/cam4.2100

14. Henke L, Kashani R, Robinson C, et al.: Phase I trial of stereotactic MR-guided online adaptive radiation therapy (SMART) for the treatment of oligometastatic or unresectable primary malignancies of the abdomen. Radiother Oncol. 2018, 126:519-526.

10.1016/j.radonc.2017.11.032 